\title{
Male infertility diagnostic laboratories during COVID-19 pandemic: development of a novel teaching/learning strategy
}

\section{Joana Santiago, Pedro O. Corda, Margarida Fardilha}

Department of Medical Sciences, Institute of Biomedicine - iBiMED, University of Aveiro, Aveiro, Portugal.

\begin{abstract}
Objective: To design and implement a laboratory teaching/learning strategy applied to male infertility diagnostic during COVID-19 pandemic.

Methods: Seventy-five students attending Module 3 (M3): Male Infertility Diagnostic Laboratories, of curricular unit Laboratories in Biomedicine 5 were divided into 8 groups to reduce students' number in class. M3 was organized in 2 Wet labs (WL; laboratory training) and 2 Dry labs (DL; theoretical-practical classes). At the end of M3, students completed a satisfaction survey.
\end{abstract}

Results: Results showed that most students were satisfied with topics addressed, time dedicated outside the class, evaluation, and overall organization of WL and DL. The protocols provided and general conditions of WL were considered satisfactory. Only positive classifications were attributed to the learning goals, relevance of knowledge acquired, and opportunities to ask about evaluation. Qualitative data suggested that the dynamics, innovation, and organization of classes, allied to the knowledge and motivation of professors, provided the appropriate learning environment.

Conclusions: The methodology implemented in M3 during COVID-19 pandemic represents a valid strategy to encourage and motivate students learning, stimulate teamwork, communication, and autonomy in the laboratory. Preparing dynamic, attractive, and motivating classes increases student's general satisfaction, improving the efficiency of the learning/teaching process.

Keywords: COVID-19; male infertility; diagnostic tools; practical classes; laboratory instruction; students' motivation. 


\section{Introduction}

As all the globe, Portugal is facing the novel coronavirus disease 2019 (COVID-19), with the academic activities suspended since the first lockdown in March 2020. Despite e-classes being very helpful during the lockdown, and the positive feedback given by the students to them (Gohiya \& Gohiya, 2020), some virtual learning activities, such as practical classes, are difficult to implement. Traditional laboratory disciplines, such as biochemistry and medical laboratory sciences were suspended during the lockdown. As laboratory practice is a valuable part of (bio)medical sciences, in which students can practice theoretical concepts learned in lecture-based classes, gain experience in the laboratory environment, and acquire basic technical and social skills for their future careers, virtual laboratories emerged as a powerful educational tool in the Era of COVID-19 (Uchejeso, Chinaza, \& Obiora, 2020; Vasiliadou, 2020). Virtual learning enables professors to demonstrate practical techniques and allows students to conduct experiments at home, using virtual platforms (Allen \& Barker, 2021; Uchejeso et al., 2020; Vasiliadou, 2020). However, this new methodology cannot replace entirely the traditional laboratory experience.

In October 2020, most educational institutions in Portugal started to re-open following strict governmental and institutional guidelines and as some of them restarted in-person classes (e.g., practical classes), some challenges had to be surpassed to assure students, faculty, and staff safety. Besides wearing personal protective equipment, as face coverings, washing hands often, and ensuring good ventilation, practicing social distancing is an important measure to reduce the spread of COVID-19 during in-person classes (Dickie \& Remusemsermann, 2020). Thus, teaching/learning methodologies needed to be restructured to follow these norms. The University of Aveiro (UA) decided to reduce the number of students attending classes and laboratory instructions that could not be delivered remotely, allowing participants to maintain social distancing and not to share objects and equipment.

The UA offers a 3-year Biomedical Sciences bachelor, that includes in the 3rd year (1st semester) the Laboratories in Biomedicine 5 (LBM5). The learning goals for LBM5 were to teach students the tools used in molecular and cellular diagnosis, allowing students to develop laboratory competencies. LBM5 is divided into 3 modules taught by researchers in the field. M3 focuses on male infertility diagnosis and usually consists of 4 practical classes in the laboratory (Wet Lab; WL), each with 3 hours duration. Due to COVID-19 contingencies, the number of students had to be reduced to half. The main objective of this study was to design and implement a new practical teaching/learning strategy applied to male infertility diagnostic in the COVID-19 pandemic and clarify if this methodology contributed to maintaining student's motivation and interest without compromising their health and safety. We intended to promote communication and teamwork skills among students during laboratory training, boosting their scientific and research knowledge and stimulating their interest for the human reproduction field. 


\section{Methods}

\subsection{Students and professors' background}

Seventy-five $3^{\text {rd }}$ year students were enrolled in M3. Students were familiar with a studentcentered learning approach, as problem-based learning is implemented in the bachelor course (Bate, Hommes, Duvivier, \& Taylor, 2014) and they all had laboratory experience from previous classes. The professors belong to the Signal Transduction Lab, Institute of Biomedicine (UA), being researchers in the male (in)fertility field. All professors had considerable laboratory experience, particularly in tasks developed throughout M3.

\subsection{Module 3 design}

With 4 weeks duration, M3 was delivered as part of the regular curriculum and focused on male infertility diagnostic. Learning objectives, content, and total contact time were not changed. The professors developed an additional component, based on a theoretical-practical approach - Dry Lab (DL) - to follow the restrictions imposed. The module design and evaluation were re-organized, comprising 2 laboratory classes (WL), and 2 theoreticalpractical classes (DL). All information concerning M3 was organized in a Padlet mural (www.padlet.com). The teaching/learning activities of M3 are integrated in Figure 1.

\begin{tabular}{|c|c|c|c|c|}
\hline \multicolumn{5}{|c|}{$1^{\text {st }}$ Meeting with the Focus Group } \\
\hline Learning approach & \multicolumn{2}{|c|}{ Wet Lab } & \multicolumn{2}{|c|}{ Dry Lab } \\
\hline Learning strategy & \multicolumn{2}{|c|}{ Practical learning in laboratory } & \multicolumn{2}{|c|}{ Theoretical/practical learning } \\
\hline Skills developed & \multicolumn{2}{|c|}{$\begin{array}{l}\text { Molecular and cellular biology techniques } \\
\text { used in male infertility diagnosis }\end{array}$} & \multicolumn{2}{|c|}{$\begin{array}{l}\text { Critical thinking; group work; } \\
\text { communication skills }\end{array}$} \\
\hline Topics covered & $\begin{array}{l}\text { WL1: } \\
\text { Basic semen } \\
\text { analysis }\end{array}$ & $\begin{array}{l}\text { WL2: } \\
\text { Evaluation of accessory } \\
\text { glands' function }\end{array}$ & $\begin{array}{c}\text { DL1: } \\
\text { Molecular markers } \\
\text { in semen }\end{array}$ & $\begin{array}{c}\text { DL2: } \\
\text { Embryo analysis by } \\
\text { deep learning }\end{array}$ \\
\hline Evaluation & $\begin{array}{l}\text { Report (12\%) } \\
\text { Peer evaluation } \\
(2 \%)\end{array}$ & $\begin{array}{l}\text { Mini-test }(12 \%) \\
\text { Peer evaluation }(2 \%)\end{array}$ & $\begin{array}{l}\text { Article review guide } \\
(12 \%)\end{array}$ & \\
\hline \multicolumn{5}{|c|}{ Students' satisfaction survey } \\
\hline \multicolumn{5}{|c|}{$2^{\text {nd }}$ Meeting with the Focus Group } \\
\hline
\end{tabular}

Figure 1. Overall organization of Module 3. Learning approaches and strategies, skills developed, topics covered, and evaluation were presented. WL, Wet lab; DL, Dry lab.

Considering that students are consumers of education, they have the right and the responsibility to be involved in curricular reform and give their feedback freely (Hsih et al., 2015). Therefore, a focus group was created for M3 conception and evaluation process, composed of 4 randomly selected students. Before M3 started, professors and the focus group met to discuss possible new approaches, topics of interest and evaluation methods. The 
students' involvement in the course preparation and organization allowed professors to adapt the learning approaches and topics addressed to the main interests of the students, improving their motivation. In the end, the professors met again with the focus group to summarize issues raised, recognize aspects to be improved in the following years, and discuss potential solutions targeting the identified opportunities for improvement.

To assure that the number of students per class did not exceed 10, the 75 students were divided into 8 groups, each one attending $2 \mathrm{WL}$ and $2 \mathrm{DL}$. The WL aimed to provide practical training in cellular and molecular diagnostic of male infertility, focusing on basic semen analysis (WL1) and molecular markers of accessory glands (WL2). The lab activities were preceded by a theoretical contextualization and during the expository part, students were encouraged to interact by asking questions. Protocols were provided at least 1 week ahead enabling the students to prepare the activity. In WL1, the protocol included the necessary steps to semen macroscopic and microscopic analysis (motility, concentration, vitality, and morphology). In WL2, the protocols were taken from the Fructose Test (FP09 I29 R01) and Citric Acid Test (FP09 I37 R01) kits to evaluate the accessory gland function, through the analysis of these molecular markers. The professors guided the students throughout the protocol steps, explaining the details associated and the technical procedures. The students were encouraged to independently think and learn with peers. During the class, students used personal protective equipment (mask, gloves, and lab coat), each pair of students had their own material, reagents, equipment, and workbench, thus maximizing the distance between stations and avoiding sharing objects. Also, the contact surfaces were frequently cleaned and disinfected, as recommended (Dickie \& Remus-emsermann, 2020). After each class students were evaluated by reports/mini questionnaires that comprised several multiple-choice questions, open questions, and data analysis related to the topics and the results obtained during the WL. Only the mini test was an individual assessment. In the end, students were asked to evaluate their peers' performance in WL - peer evaluation. This evaluation tool aimed to determine the quality of teamwork, being an individual reflection of students' dedication, working, and communication.

The DLs were mainly based on scientific papers and imaging analysis, providing to students the scientific tools, methodologies, and soft skills required to work in a clinical or research laboratory in the male reproductive field. In DL1, focused on identifying molecular markers in semen, the students were challenged to analyze papers using the platform Perusall (https://perusall.com/). Perusall allows students to read, generate comments and discuss ideas with other colleagues, creating a virtual scientific discussion that stimulates their critical thinking, written communication, and group work. A guide for the analysis of the paper was provided by the professor to be filled in pairs as an evaluation exercise. In DL2, the students became familiar with embryo analysis using deep learning. Due to the novelty of the topic, several research papers were provided to the students before the class for their autonomous 
preparation. During the class, an introduction to the fundamental concepts of deep learning and how this technology is being applied to in vitro fertilization was made. Then, a hypothetical problem was provided to students that had to design a putative workflow for analyzing embryo images using this tool. Finally, a class discussion was held for the different pairs to share their work and for the professor to give tips on the best approach to take about the proposed problem. The main objective of DL2 was to improve the students' ability to identify a study question, to analyze critically scientific information, and to build protocols to be applied in the research context.

\subsection{Data collection and analysis of the survey}

As a quality control exercise, students fill out a structured questionnaire classifying the teaching/learning experience. To generate a comprehensive picture of the students' perspectives, the survey combines a quantitative and qualitative approach. Each topic to be quantitatively assessed by the student included several questions related to that issue, rated on a Likert scale (1-7). Answers of " 1 " indicate the most negative response (Completely dissatisfied), while "7" the most positive (Completely satisfied). We also asked students to identify 2 positive aspects, 2 aspects that need to be improved, and what they liked most in M3. To assure student's anonymity no personal data were collected.

\section{Results and discussion}

To the professors, more fundamental than the student's grades was to understand if the new design of M3 fulfills students' expectations. Therefore, the results presented in this article focus not only on students' performance but also on their opinion and satisfaction about several aspects of M3 (Figure 2) and its importance to their future.

\subsection{Students' performance}

All students were able to fulfill the tasks proposed in each component. The average grade of M3 was 16.9 (from a 0 to 20 scale) with 38 students $(52 \%)$ having classifications $\geq 17.0$. Only 6 students were graded $<15.0$ and only one student performed poorly (10.7). In peer evaluation, the average grade of students was 18.6 (from a 0 to 20 scale) with 53 students (72.6\%) having classification higher than 18.7 . Only 2 students were graded $\leq 15$, and 1 student was not classified. The article review guide (DL1) allowed us to access the impact of DL on soft skills development (critical thinking and effective communication).

\subsection{Survey analysis}

From the 75 students that participated in M3 in the curricular year 2020/2021, 4\% (n=3) did not answer the questionnaires. 


\subsubsection{Quantitative results}

Quantitative data analysis showed that most students were satisfied with the topics addressed (99\%), protocols provided (97\%), general conditions existent in the lab (96\%), including the material and reagents available, contribution/relevance for their education (96\%) and overall organization (99\%) of WL (Figure 2.A). Concerning evaluation and time dispended outside class, $4 \%$ and $3 \%$ of the students are somewhat dissatisfied, respectively.
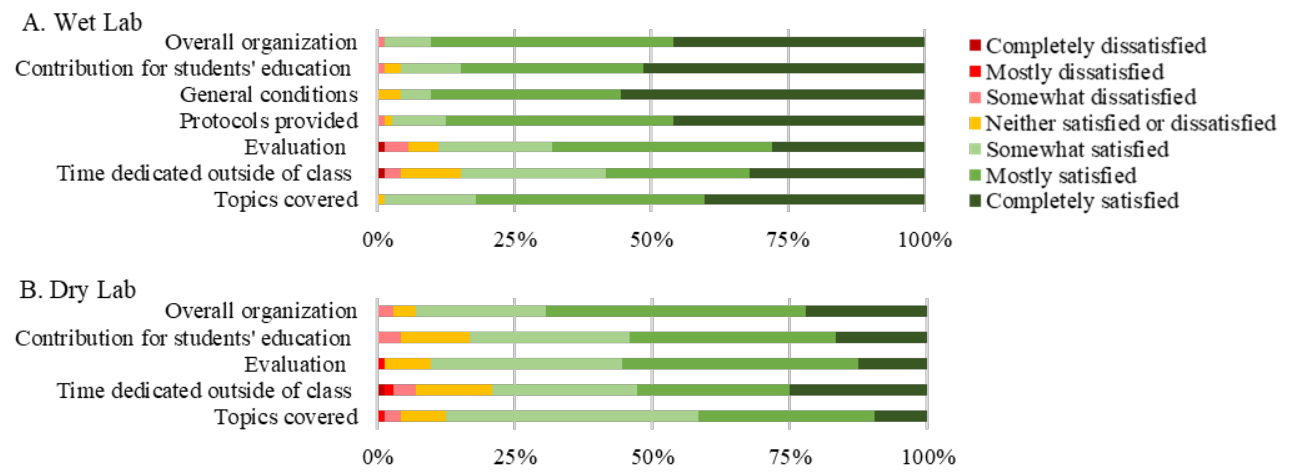

C. Module 3

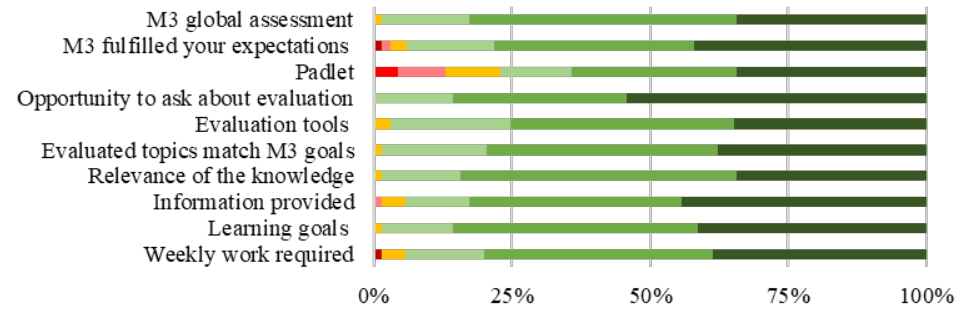

Figure 2. Students' evaluation of (A) Wet and (B) Dry Labs, and (C) Module 3.

The DL (Figure 2.B) received more negative evaluations than the WL. Some students were dissatisfied or neither satisfied nor dissatisfied with the time dedicated outside of class $(21 \%)$, with the contribution for their education (17\%), and with the topics covered $(12 \%)$. Nevertheless, they attributed predominantly positive classifications to all the questions. Generally, most students agreed that the weekly work required was appropriate $(94 \%)$, the module learning goals were unambiguously provided (99\%), important information was provided in time (94\%), the practical and theoretical knowledge acquired was relevant for their education (99\%), and the evaluated topics correspond to the previously stated goals (97\%) (Figure 2.C). All students were satisfied with the opportunities to ask about how the evaluation was performed. Overall, the module fulfilled the expectations of $94 \%$ of the students, with $99 \%$ of them satisfied with M3. Finally, regarding professors, all students are somewhat, mostly, or completely satisfied with their motivation, knowledge on the topics covered, availability to answer questions, and global performance. 


\subsubsection{Qualitative results}

Students were asked to indicate 2 positive and 2 aspects that could be improved. They indicated that the contents covered in classes were interesting, actual, relevant, and the necessary knowledge bases were provided (21 students). Almost $30 \%$ of students indicated as a positive aspect of the module the professors, particularly their knowledge on the topics addressed, motivation, enthusiasm, clarity, and availability to help students and answer their questions. The students also considered that the variety of methodologies used increased the dynamic of the classes stimulating their interest, motivation, and concentration. They also enjoy work in groups and the analysis of scientific papers that improve their critical thinking. Regarding student's views on ways to improve teaching and M3, 26 students did not answer or indicated that they had nothing to point out. The aspect that most students think needs to be improved was the DL (contents covered, time required for preparation). The platforms used (Padlet and Perusall) were recognized as aspects to improve. Some students considered difficult to find information in Padlet and suggested accessing it through the University elearning platform. Concerning Perusall, the students mention they need more time to explore all its functionalities.

Regarding what students like the most: the WL, as well as the topics covered, considering infertility an interesting and captivating topic; the availability, motivation, and interest of the professors that motivate the students for the classes and the organization of the module were also the aspects more appreciated by students. Quoting some of the students' comments about M3, "Congratulations, the module is really well designed and will certainly benefit the future students"; "Congratulations to the professors, a module taught with enthusiasm is very motivating for those who are learning"; "The environment provided by the teachers in the laboratory was very good, an environment where you can work and learn well". These data suggested that the approach adopted represents a valid and enjoyable strategy to encourage, captivate and motivate students to learn about male infertility, stimulate teamwork, communication, and independence in the laboratory.

\section{Conclusion}

Results showed that the dynamics, innovation, and organization of the classes, allied to the knowledge, motivation, and availability of the professors, provided the appropriate environment to learn. However, the lack of data from students attending the traditional methodology does not allow the comparison of student's satisfaction before and after the implementation of this methodology, which represents the major limitation of this study. To minimize the contacts and guarantee compliance with social distance without compromising students' interaction, cooperation, group work, and discussion of ideas, students were divided into pairs that remained throughout the module. Thus, we were able to promote teamwork, social interaction and collaboration among the students maintaining their safety. The focus 
group was of great importance, since it allowed students to contribute and shape their education, increasing their motivation and engagement. Investing in preparing attractive and motivating classes increases students' general satisfaction and the learning/teaching process becomes more efficient. The positive results presented should encourage others in a similar situation to follow this approach.

\section{Acknowledgments}

The authors thank the DCM, UA for the infrastructure support, and the support from FCT of the Portuguese Ministry of Science and Higher Education to J.S. (SFRH/BD/136896/2018) and P.C. (2020.10111.BD). The authors deeply acknowledge the students of the curricular unit Laboratories of Biomedicine 5 of the Biomedical Sciences degree of the UA (class 2020/2021) for being highly motivated and for motivating the professors.

\section{References}

Allen, T. E., \& Barker, S. D. (2021). BME Labs in the Era of COVID-19: Transitioning a Hands-on Integrative Lab Experience to Remote Instruction Using Gamified Lab Simulations. Biomedical Engineering Education, 1(1), 99-104. doi: 10.1007/s43683020-00015-y

Bate, E., Hommes, J., Duvivier, R., \& Taylor, D. C. M. (2014). Problem-based learning (PBL): Getting the most out of your students - Their roles and responsibilities: AMEE Guide No. 84. Medical Teacher, 36(1), 1-12. doi: 10.3109/0142159X.2014.848269

Dickie, I. A., \& Remus-emsermann, M. (2020). Teaching laboratory best practice considerations for Covid19. (April).

Gohiya, P., \& Gohiya, A. (2020). E -learning during Covid 19 Pandemic. Research Square, 4-9. doi: 10.21203/rs.3.rs-29575/v1

Hsih, K. W., Iscoe, M. S., Lupton, J. R., Mains, T. E., Nayar, S. K., Orlando, M. S., Goldberg, H. R. (2015). The Student Curriculum Review Team: How we catalyze curricular changes through a student-centered approach. Medical Teacher, 37(11), 1008-1012. doi: 10.3109/0142159X.2014.990877

Uchejeso, O. M., Chinaza, I. R., \& Obiora, E. R. (2020). COVID-19: The stimulus for virtual learning in Medical Laboratory Science. Journal of Bio Innovation, 9(5), 812-820. doi: 10.46344/JBINO.2020.v09i05.17

Vasiliadou, R. (2020). Virtual laboratories during coronavirus (COVID-19) pandemic. Biochemistry and Molecular Biology Education: A Bimonthly Publication of the International Union of Biochemistry and Molecular Biology, 48(5), 482-483. doi: 10.1002/bmb.21407 